\title{
C4d/CD34 double-immunofluorescence staining of renal allograft biopsies for assessing peritubular capillary C4d positivity
}

\author{
Kuang-Yu Jen ${ }^{1}$, Thuy B Nguyen ${ }^{1}$, Flavio G Vincenti ${ }^{2}$ and Zoltan G Laszik ${ }^{1}$ \\ ${ }^{1}$ Department of Pathology, University of California, San Francisco, San Francisco, CA, USA and \\ ${ }^{2}$ Kidney Transplant Service, University of California, San Francisco, San Francisco, CA, USA
}

\begin{abstract}
Immunofluorescence detection of the complement split product C4d along peritubular capillaries in renal allograft biopsies is the mainstay for the diagnosis of antibody-mediated rejection. The extent of peritubular capillary C4d positivity may have significant clinical ramifications; however, peritubular capillary density in the renal cortex is often difficult to assess with single-channel immunofluorescence. In this study, we report a C4d/CD34 double-immunofluorescence staining protocol for renal allograft frozen sections that allows rapid and sensitive detection of C4d positivity, as well as improved accuracy in estimating the C4d-positive fraction of peritubular capillaries. In addition, this method aids in determining whether C4d-positive structures correspond to peritubular capillaries or whether they represent common mimics of peritubular capillaries such as tubular basement membranes. C4d/CD34 double immunofluorescence provides rapid, convenient, and low-cost implementation for laboratories currently utilizing single-channel C4d immunofluorescence.
\end{abstract}

Modern Pathology (2012) 25, 434-438; doi:10.1038/modpathol.2011.168; published online 28 October 2011

Keywords: antibody mediated; CD34; C4d; immunofluorescence; peritubular capillaries; rejection

Positive immunostaining for the complement split product C4d within peritubular capillaries is critical for the diagnosis of antibody-mediated rejection in renal allograft biopsies. Diffuse C4d positivity, defined as $>50 \%$ peritubular capillary involvement, is highly correlated to the presence of circulating donor-specific antibodies and is required by the Banff 07 Classification of Renal Allograft Pathology for the diagnosis of acute and chronic antibody-mediated rejection. ${ }^{1}$ However, the clinical significance of focal $(<50 \%)$ peritubular capillary C4d positivity remains unclear. Some studies suggest that even focal C4d staining may have significant clinical ramifications, while other studies fail to show such outcomes. ${ }^{2-5}$ Nevertheless, the data are limited and further evaluation with regard to long-term graft survival is needed.

Correspondence: Dr ZG Laszik, MD, PhD, Department of Pathology, University of California, San Francisco, 513 Parnassus Avenue, San Francisco, CA 94143, USA.

E-mail: Zoltan.Laszik@ucsfmedctr.org

Received 25 March 2011; revised 7 September 2011; accepted 7 September 2011; published online 28 October 2011
In order to make any headway into tackling this issue, a reliable method for assessing the extent of C4d-positive peritubular capillaries is essential. Currently, indirect immunofluorescence staining with anti-C4d antibody on frozen sections of fresh/ unfixed tissues is the most widely used method and offers slightly higher sensitivity than immunohistochemical stains. ${ }^{6-8}$ Although the fraction of C4dpositive peritubular capillaries can be visually estimated using this method, it is not uncommon to come across situations where single-channel C4d immunofluorescence is extremely difficult to interpret. This obstacle is especially evident in biopsies with significant interstitial fibrosis and tubular atrophy where the density of peritubular capillaries is uncertain. Furthermore, positive C4d signal in other structures, such as tubular basement membranes, may mimic peritubular capillaries. Therefore, an accurate and reproducible method for assessing the percentage of peritubular capillary C4d positivity is greatly needed.

In this study, we improve on single-channel C4d immunofluorescence by using $\mathrm{C} 4 \mathrm{~d}$ in combination with the vascular endothelial marker CD34 to implement a C4d/CD34 double-immunofluorescence 
staining protocol for frozen tissue. This method of dual-channel immunofluorescence evaluation not only allows for rapid and sensitive detection of C4d positivity in renal allograft biopsies, but it also provides improved accuracy when assessing the extent of C4d-positive peritubular capillaries.

\section{Materials and methods}

Seventeen renal allograft biopsies with positive peritubular capillary C4d staining, as determined by single-channel C4d immunofluorescence on frozen sections, were used for the study. The biopsies were chosen to represent a range of positive C4d intensities and extent of peritubular capillary staining as reported previously in the diagnostic pathology report. Four renal allograft biopsies negative for C4d immunofluorescence were used as negative controls.

Four-micrometer-thick frozen sections of the biopsies were fixed in acetone for $5 \mathrm{~min}$ at $-20^{\circ} \mathrm{C}$ and incubated with a mixture of polyclonal rabbit anti-C4d (ALPCO, Salem, NH, USA) and mouse monoclonal anti-CD34 (DAKO, Carpinteria, CA, USA) antibodies for $60 \mathrm{~min}$ at a dilution of 1:50 and 1:100, respectively. Subsequently, the slides were incubated with a mixture of fluorescein isothiocyanate (FITC)-labeled goat anti-rabbit (Vector, Burlingame, CA, USA) and Texas Redlabeled horse anti-mouse (Vector) antibodies for $30 \mathrm{~min}$. Both secondary antibodies were used at a dilution of 1:100. All incubations were performed in a humidified chamber in the dark at room temperature.

C4d/CD34 double-immunofluorescence frozen section slides were visually examined under immunofluorescence microscopy using an Olympus BX41 fluorescence microscope (Olympus, Center Valley, PA, USA), and digital images were captured using an attached Olympus DP71 digital camera with the $\times 20$ objective. Multiple non-overlapping fields were captured in order to provide full coverage of the entire biopsy tissue. For each field, two images were captured, one using a green filter for FITC fluorescence and one using a red filter for Texas Red fluorescence. The digital images were saved as 24-bit RGB TIFF files with a final resolution of $2040 \times 1536$ pixels. These files were imported into the freely available image processing computer program ImageJ, converted to 8-bit grayscale images, and background subtraction was performed using a 'rolling ball radius' of 50 pixels and with the 'sliding parabaloid' function set to on (National Institutes of Health, Bethesda, MD, USA, http://imagej.nih. gov/ij/). Next, glomeruli and large vessels were manually masked. Subsequently, a pixel intensity threshold was automatically set using the built-in 'moments' thresholding method. Finally, each processed image for the C4d/FITC green channel along with its corresponding CD34/Texas Red channel was imported into the ImageJ plugin Just Another Colocalization Plugin (JACoP) to calculate Manders coefficient M2. ${ }^{9}$

\section{Results}

Visual examination of C4d/CD34 double-immunofluorescence sections revealed excellent signal with low background in both channels. These sections exhibited comparable C4d intensities as sections stained for C4d alone (Table 1). The CD34 immunostain highlighted peritubular capillaries and allowed determination of overall peritubular capillary density. This channel also provided a basis of comparison for estimating the percentage of C4dpositive peritubular capillaries.

A common diagnostic dilemma encountered in routine single channel C4d immunofluorescence is differentiating between positive signals in tubular basement membranes versus dilated peritubular capillaries. C4d/CD34 double immunofluorescence helps resolve this problem. Positive C4d staining in tubular basement membranes does not colocalize with CD34-positive peritubular capillaries, while co-localization of the C4d and CD34 signals indicates peritubular capillary C4d positivity (Figure 1a).

Consistent with single-channel C4d immunofluorescence results, several of the biopsies exhibited focal C4d positivity on C4d/CD34 double immunofluorescence (Figure 1b). The percentage of C4dpositive peritubular capillary was visually estimated for each case using C4d/CD34 double immunofluorescence and compared with the results from singlechannel C4d immunofluorescence. In most cases, estimated peritubular capillary C4d extent was comparable for the two methods; however, a few cases showed dramatic differences (Table 1). The disagreement arose from inaccurate assessment of overall peritubular capillary density with singlechannel C4d immunofluorescence. With the aid of the CD34 channel, accuracy for the extent of peritubular capillary C4d staining by visual estimation was improved. Of note, the extent of peritubular capillary C4d staining demonstrated no correlation with the degree of chronicity (interstitial fibrosis and tubular atrophy) or concurrent pathology seen in the corresponding paraffin-embedded tissue for each case (Table 1).

As C4d/CD34 double immunofluorescence exhibits strong signal with low background, computerassisted morphometric analysis to calculate the extent of C4d positivity in peritubular capillaries is also applicable. Using the freely available image analysis program ImageJ, the green and red channel immunofluorescence images corresponding to the C4d and CD34 immunostains, respectively, were processed, overlaid, and the Manders coefficients (M2) were calculated (Figure 2 and Table 1). 
Table 1 Comparison of various methodologies for the assessment of C4d positivity in peritubular capillaries

\begin{tabular}{|c|c|c|c|c|c|c|c|}
\hline \multirow[t]{2}{*}{ Case } & \multicolumn{2}{|c|}{$C 4 d / C D 34 I F$} & \multicolumn{2}{|c|}{$C 4 d I F$} & \multirow[t]{2}{*}{$M 2^{\mathrm{a}}$} & \multirow[t]{2}{*}{ Additional diagnosis ${ }^{\mathrm{b}}$} & \multirow[t]{2}{*}{$I F / T A^{\circ}$} \\
\hline & Intensity $^{\mathrm{d}}$ & Extent $(\%)^{\mathrm{d}}$ & Intensity ${ }^{\mathrm{d}}$ & Extent $(\%)^{\mathrm{d}}$ & & & \\
\hline 1 & $0+$ & 0 & $0+$ & 0 & 0.01 & Normal & 0 \\
\hline 2 & $0+$ & 0 & $0+$ & 0 & 0.01 & Normal & 0 \\
\hline 3 & $0+$ & 0 & $0+$ & 0 & 0.01 & Normal & 0 \\
\hline 4 & $1+$ & 5 & $0+$ & 0 & 0.02 & Normal & 0 \\
\hline 5 & $2+$ & 5 & $2+$ & $<10$ & 0.03 & ATMR (IIA) & 0 \\
\hline 6 & $1+$ & 10 & $1+$ & $10-50$ & 0.08 & TxAP & 1 \\
\hline 7 & $2+$ & 10 & $3+$ & $10-50$ & 0.09 & TxGP & 0 \\
\hline 8 & $1+$ & 30 & $1+$ & $10-50$ & 0.42 & TxGP & 3 \\
\hline 9 & $1-2+$ & 40 & $1+$ & $<10$ & 0.49 & ATMR (IA) & 0 \\
\hline 10 & $2+$ & 50 & $3+$ & $>50$ & 0.40 & ATMR (IA) & 0 \\
\hline 11 & $3+$ & 50 & $3+$ & $>50$ & 0.28 & ATMR (IA) and TxGP & 0 \\
\hline 12 & $2+$ & 60 & $2+$ & $10-50$ & 0.48 & ATMR (IA) & 0 \\
\hline 13 & $2+$ & 60 & $1+$ & $>50$ & 0.40 & No ATMR & 0 \\
\hline 14 & $1+$ & 60 & $1-2+$ & $>50$ & 0.60 & TMA & 0 \\
\hline 15 & $2+$ & 60 & $2+$ & $>50$ & 0.55 & TxGP & 1 \\
\hline 16 & $2+$ & 60 & $3+$ & $>50$ & 0.51 & Borderline & 1 \\
\hline 17 & $2+$ & 70 & $2+$ & $>50$ & 0.49 & No ATMR & 3 \\
\hline 18 & $3+$ & 80 & $3+$ & $>50$ & 0.59 & TxGP & 2 \\
\hline 19 & $3+$ & 90 & $2+$ & $>50$ & 0.60 & ATMR (IIA) & 1 \\
\hline 20 & $3+$ & 100 & $3+$ & $>50$ & 0.75 & ATMR (IA) & 0 \\
\hline 21 & $3+$ & 100 & $3+$ & $>50$ & 0.73 & TxGP & 1 \\
\hline
\end{tabular}

ATMR, acute T-cell-mediated rejection; TxAP, transplant arteriopathy; TxGP, transplant glomerulopathy; TMA, thrombotic microangiopathy. ${ }^{\mathrm{a}}$ The fraction of CD34-positive pixels that overlap/co-localize with C4d-positive pixels is morphometrically calculated from the C4d/CD34 double-stained sections (M2, Manders coefficient).

${ }^{\mathrm{b}}$ Other concurrent pathology seen on the biopsy specimen based on paraffin-embedded tissue. Rejection classification based on Banff 07 Classification of Renal Allograft Pathology.

${ }^{\mathrm{C}} \mathrm{IF} / \mathrm{TA}$ (interstitial fibrosis and tubular atrophy) scores are indicated as none (0), mild (1), moderate (2) and severe (3).

${ }^{\mathrm{d}}$ Reported for C4d positivity from visual examination of C4d/CD34 double-stained sections (C4d/CD34 IF) and C4d single-stained sections (C4d IF). Intensity is graded from $0+$ (negative) to $3+$ (bright) and extent is provided as percentage of C4d-positive peritubular capillaries.

M2 represents the fraction of CD34-positive pixels that overlap/co-localize with C4d-positive pixels and ranges from 0 to 1 . A high $\mathrm{M} 2$ value indicates substantial co-localization of the two channels, whereas lower M2 values represent a lesser degree of co-localization. M2 values determined from morphometric evaluation correlated well with visual evaluation of C4d/CD34 double-immunofluorescence-stained sections (Figure 3).

\section{Discussion}

Standard single-channel C4d immunofluorescence on frozen sections has inherent disadvantages that limit accurate assessment of positive signal in peritubular capillaries. Apparent 'focal' staining using this standard method likely constitutes one of two situations: (1) true focal staining in which only a portion of the peritubular capillaries show C4d positivity or (2) diffuse peritubular capillary C4d positivity with focal loss of peritubular capillaries in the renal cortex. Distinguishing between these two possibilities is especially difficult when the distribution of peritubular capillary density is uncertain, as is often the case in biopsies with tubulointerstitial chronicity. In addition, positive signal in dilated peritubular capillaries and tubular basement membranes often mimic one another, further complicating the interpretation of singlechannel C4d immunofluorescence results. The implementation of concurrent CD34 immunostain offers a simple solution to these problems by highlighting the peritubular capillaries within the renal parenchyma and establishing a basis of comparison for C4d-positive structures.

A simple computer-assisted morphometric quantitation protocol can be applied to the images captured from the C4d and CD34 immunofluorescence channels to calculate the fraction of C4d and CD34 co-localization. Our results indicate that M2 calculated by the JACoP plugin for ImageJ is comparable to visual estimation; however, M2 is generally slightly lower than the expected fraction of C4d/CD34 co-localization based on visual assessment. The reason for this difference is that this program uses a pixel-to-pixel comparison between the two channels when determining the degree of co-localization. Minor signal bleed into adjacent pixels on brighter stains can contribute to slight pixel non-overlap between channels, resulting in lower M2. However, M2 shows excellent correlation to visual estimation and may provide a more consistent and reproducible quantitative method 
a

PTC staining

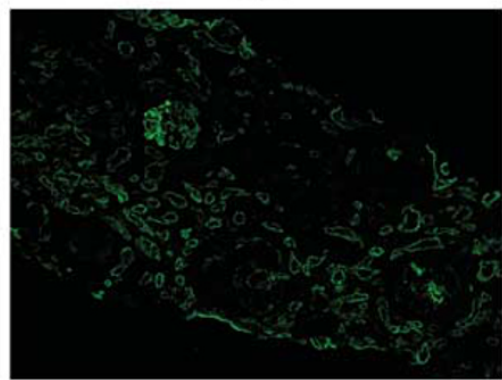

TBM staining

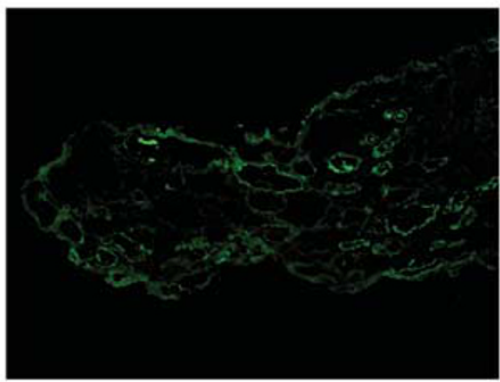

b
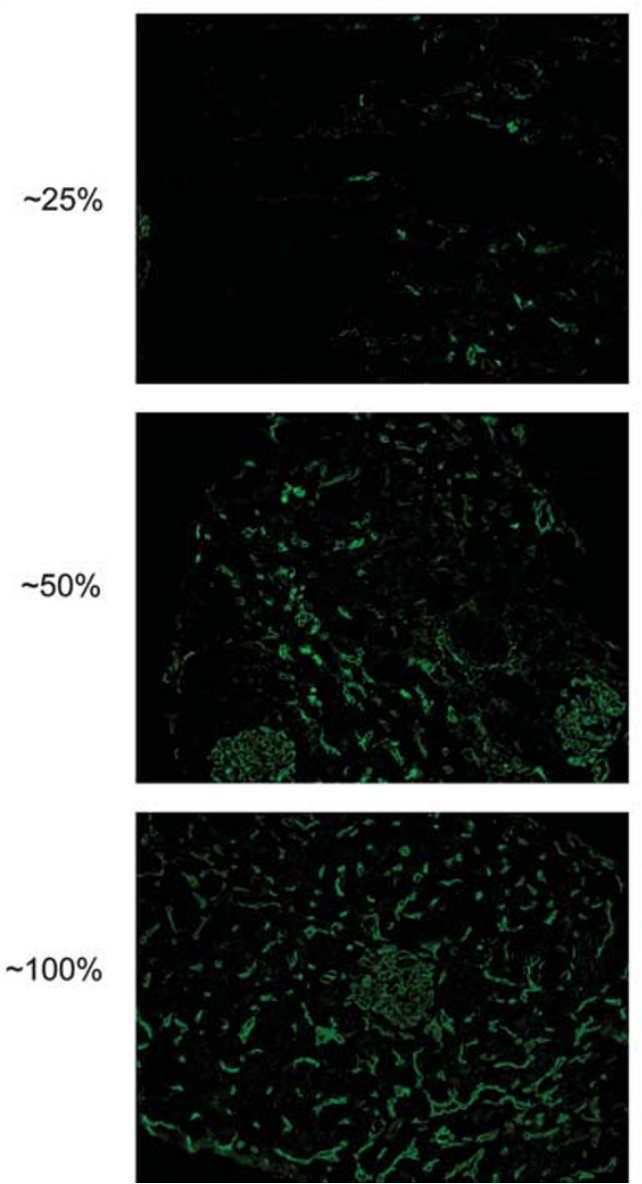

CD34
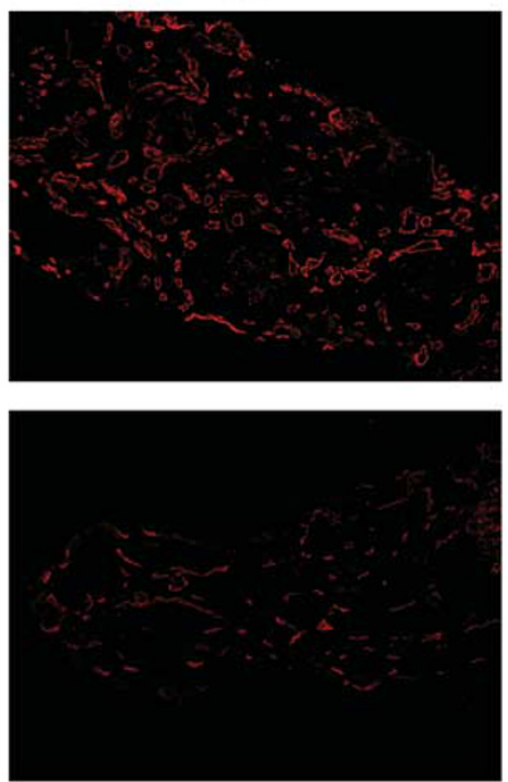

CD34
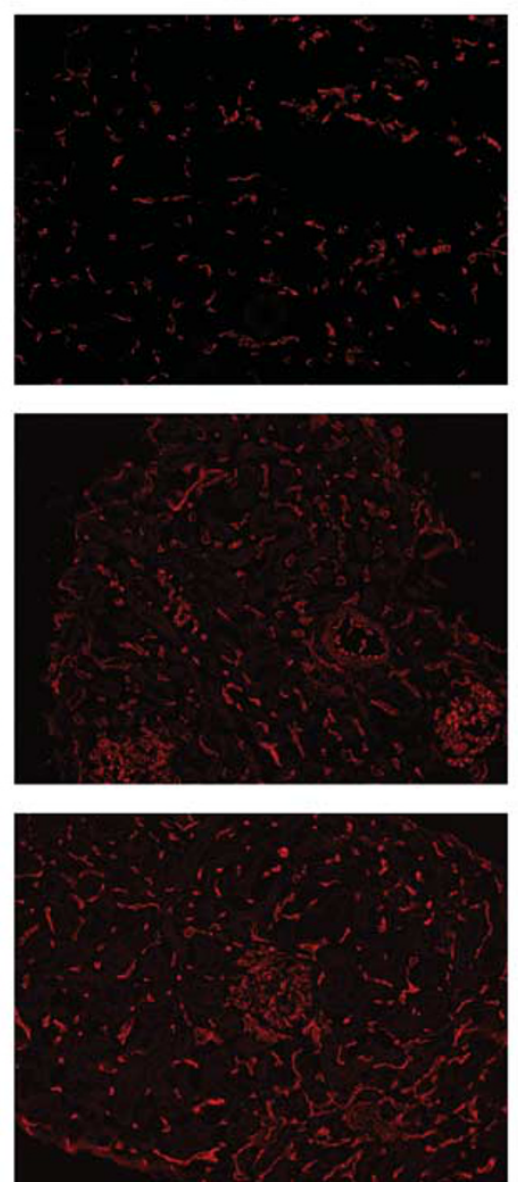

Overlay
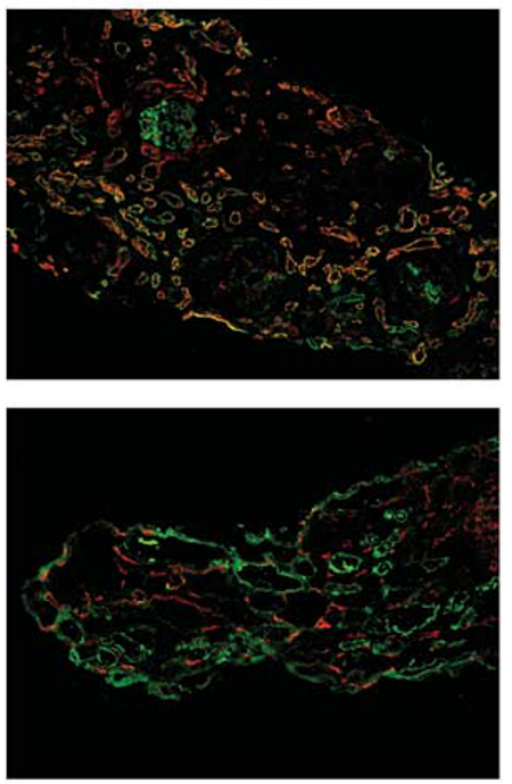

Overlay
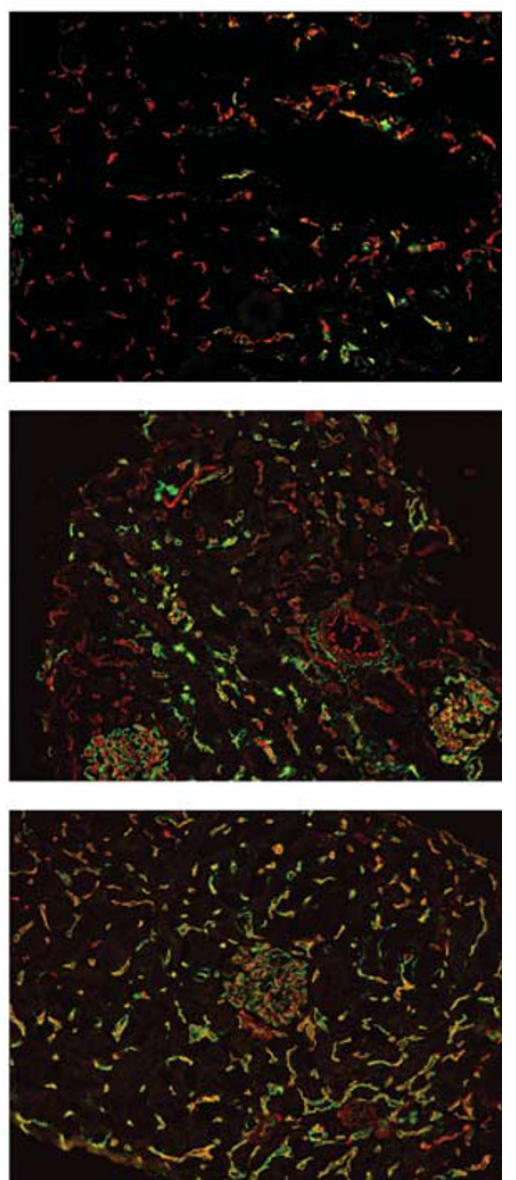

Figure 1 C4d and CD34 immunofluorescence co-localization. (a) Dilated peritubular capillaries with C4d positivity may mimic staining of tubular basement membranes of atrophic tubules (top row, left image). Using CD34 as a basis of comparison (top row, center image), co-localization of C4d and CD34 confirms C4d in peritubular capillaries (top row, right image). Tubular basement membrane C4d positivity is confirmed by the lack of CD34 co-localization (bottom row). (b) Examples of C4d (green), CD34 (red) and co-localized (overlay) staining showing extent of C4d-positive peritubular capillaries. Approximate percentages of C4d-positive peritubular capillaries by visual inspection are shown to the left. 

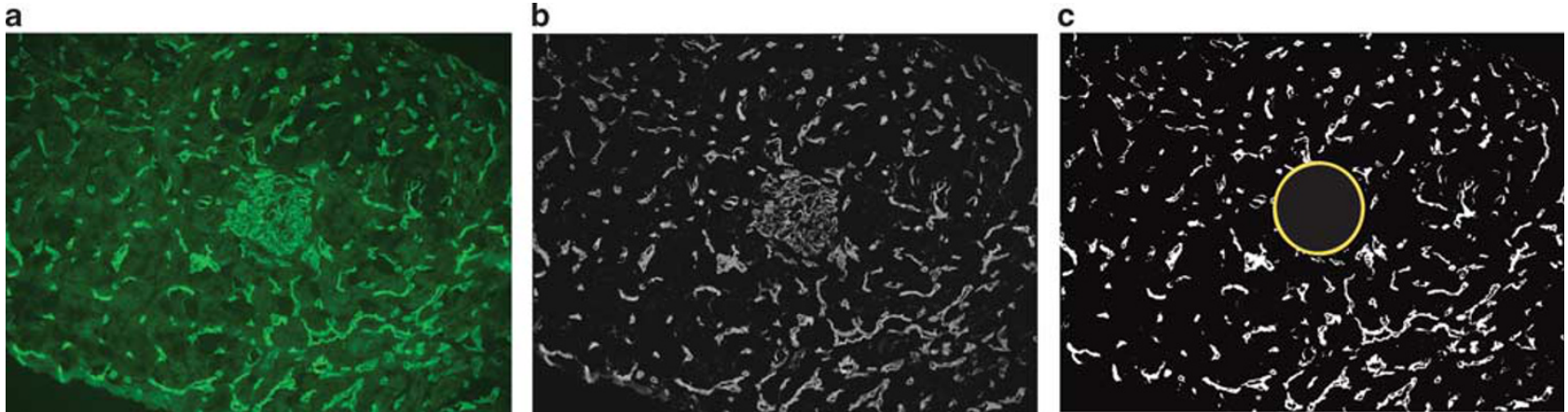

Figure 2 Image processing for computer-assisted morphometric evaluation of immunofluorescence signal. The original image of the green channel representing C4d immunofluorescence (a) is converted to a grayscale image, and the built-in ImageJ function for background subtraction is applied (b). Subsequently, the threshold for determining positive signal is applied and the glomeruli are masked (c, yellow circle) to produce the final processed image (c) for co-localization evaluation using the JACoP plugin for ImageJ.

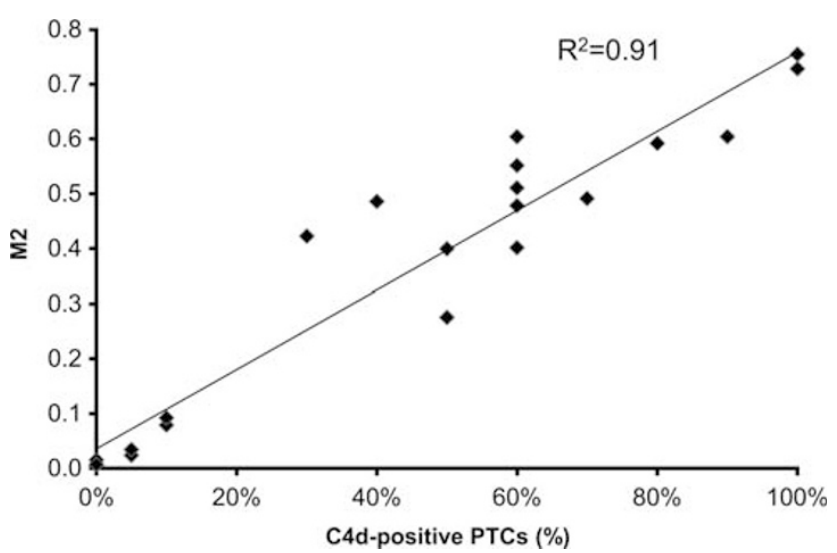

Figure 3 Computer-assisted morphometric quantitation versus visual estimation of the extent of peritubular capillary C4d positivity using C4d/CD34 double immunofluorescence. $X$-axis displays values for visual estimation. $Y$-axis indicates M2 values as determined by morphometric quantitation and represents the fraction of CD34-positive pixels with co-localization of C4dpositive pixels. Linear regression was performed and the $R^{2}$ is indicated.

for assessing the fraction of positive C4d peritubular capillaries.

In summary, C4d/CD34 double immunofluorescence is a useful yet low-cost and convenient method for C4d detection in renal allograft biopsies. It requires minimal optimization and no additional staining time. The CD34 antibody is simply mixed with the C4d antibody, as are the secondary fluorophore-labeled antibodies for each channel. Thus, laboratories currently using single-channel C4d immunofluorescence can easily implement this technique.

\section{Disclosure/conflict of interest}

The authors declare no conflict of interest.

\section{References}

1 Solez K, Colvin RB, Racusen LC, et al. Banff 07 classification of renal allograft pathology: updates and future directions. Am J Transplant 2008;8:753-760.

2 Kayler LK, Kiss L, Sharma V, et al. Acute renal allograft rejection: diagnostic significance of focal peritubular capillary C4d. Transplantation 2008;85: 813-820.

3 Magil AB, Tinckam KJ. Focal peritubular capillary C4d deposition in acute rejection. Nephrol Dial Transplant 2006;21:1382-1388.

4 Kedainis RL, Koch MJ, Brennan DC, et al. Focal C4d+ in renal allografts is associated with the presence of donorspecific antibodies and decreased allograft survival. Am J Transplant 2009;9:812-819.

5 Haririan A, Kiangkitiwan B, Kukuruga D, et al. The impact of c4d pattern and donor-specific antibody on graft survival in recipients requiring indication renal allograft biopsy. Am J Transplant 2009;9:2758-2767.

6 Nadasdy GM, Bott C, Cowden D, et al. Comparative study for the detection of peritubular capillary C4d deposition in human renal allografts using different methodologies. Hum Pathol 2005;36:1178-1185.

7 Seemayer CA, Gaspert A, Nickeleit V, et al. C4d staining of renal allograft biopsies: a comparative analysis of different staining techniques. Nephrol Dial Transplant 2007;22:568-576.

8 Troxell ML, Weintraub LA, Higgins JP, et al. Comparison of C4d immunostaining methods in renal allograft biopsies. Clin J Am Soc Nephrol 2006;1:583-591.

9 Bolte S, Cordelieres FP. A guided tour into subcellular colocalization analysis in light microscopy. J Microsc 2006;224:213-232. 